\title{
MODELING AND ANALYSIS OF BIAS-PLY MOTORCYCLE TIRES
}

\author{
YoshinoRI WATANABE $\dagger$ and Movses J. KaLDJian $\ddagger$ \\ The University of Michigan, Ann Arbor, MI 48109, U.S.A.
}

\begin{abstract}
Physical properties of motorcycle tire structures are considered to help sclect a good and true-to-life mechanistic model suitable for finite element analysis of tires. Rubber and bias-ply layered-cord together make the inflated unhomogeneous tire structure anisotropic and geometrically highly nonlinear.

A mathematical model made of 3-D solid and truss elements is discussed, and presented as realistic and adequate to accommodate the anisotropy and nonlinearity inherent in the tire problem.

Various loading cases, including a cambered tire with inflation pressure and vertical load on contact patch, are studied and evaluated for force and moment acting on the tire. The results are very encouraging and satisfactory.
\end{abstract}

\section{INTRODUCTION}

The carcass and the tread are two of the major portions that constitute a tire. The tread is the portion that is in contact with the road surface. It forms a contact patch to support the vertical loads, and governs the lateral slip and adhesion against the road surface. The configurations of tread are usually periodically axisymmetric, i.e. the same pattern recurs at circumferential intervals. The mechanical properties of tread will inevitably affect the deformation of the carcass to some extent. However, in this study, the mathematical model will not include the tread for simplicity.

In the tire deformation analysis, the tire carcass is often treated as a homogeneous anisotropic shell of revolution and local homogenuity is assumed. In such cases, the individual plies and cords are not depicted any more. The anisotropic properties of the cordrubber laminae or laminates can be calculated by techniques developed in mechanics of composite materials and the tire carcass behavior expressed by shell theory. However, in reality, a tire carcass is made of finite number of plies and is not homogeneous. Also, the cords are not invisibly small in size nor countless in number.

\section{A MECHANISTIC MODEL OF MOTORCYCLE TIRE} STRUCTURE

An approach to the tire deformation analysis using a mathematical model in which the nature of the discrete constituents is preserved is considered to be an intuitive and a more realistic worthwhile attempt. Hence, in this study, a finite element model for tire carcass (Fig. 1) built as an aggregate of finite number of cords and rubber slabs is proposed.

This model offers a great advantage in stiffness formulation. The global force-deformation response analysis can be efficiently pursued without the cum-

†Honda Motor Co. and Doctoral Student, Dept. of Mechanical Engineering.

\$Professor, Dept. of Naval Architecture and Marine Engineering. bersome recalculation of geometry related nonlinear constitutive laws necessary for the equivalent shell element model.

The element marked " $A$ " in Fig. 1, is a 3-dimensional solid element of linear, low modulus, isotropic, nearly incompressible material, representing the rubber matrix in the side-wall or in the tread. The Mooney-Rivlin material will be a more adequate representation for rubber, when strains and strain rates are large, however, the range of strain assumed in this study is small enough to justify use of lincar isotropic and nearly incompressible material.

The element marked " $B$ " also in Fig. 1, is a linear elastic truss element, representing the cords in the side-wall and the tread. This element carries tension and compression but offers no resistance to bending. The real cords are yarned fibers and have anisotropic and nonhomogeneous properties. They can carry fairly large tension but very little compression and bending. As long as the solidity in axial stiffness and the smallness of flexural rigidity are concerned, the linear elastic truss element is considered to be an adequate representation of the real cords which are pre-tensioned due to inflation pressure. Neglecting the effects of unhomogeneity, anisotropy, and low compressive modulus will produce no significant errors in the tire analysis envisaged in this research.

There are two disadvantages to this mathematical mode. The one occurs when the 3-dimensional solid elements representing the rubber matrix, and the truss elements representing the cords are joined. In the real cord-rubber lamina the bonding between the surface of cords and the surrounding rubber is probably at least more than $60 \%$, as is often assumed, whereas in the present modeling, the cords are bonded only at their ends to the 3-dimensional solid elements at the diagonal corners. This discrepancy remains to exist as long as such discretization is employed. Reducing the size of the element may improve the results somewhat but will not eliminate it completely.

The other disadvantage stems from the coarseness of element size and the limited number of cord layers that can be accommodated with the present model. In 


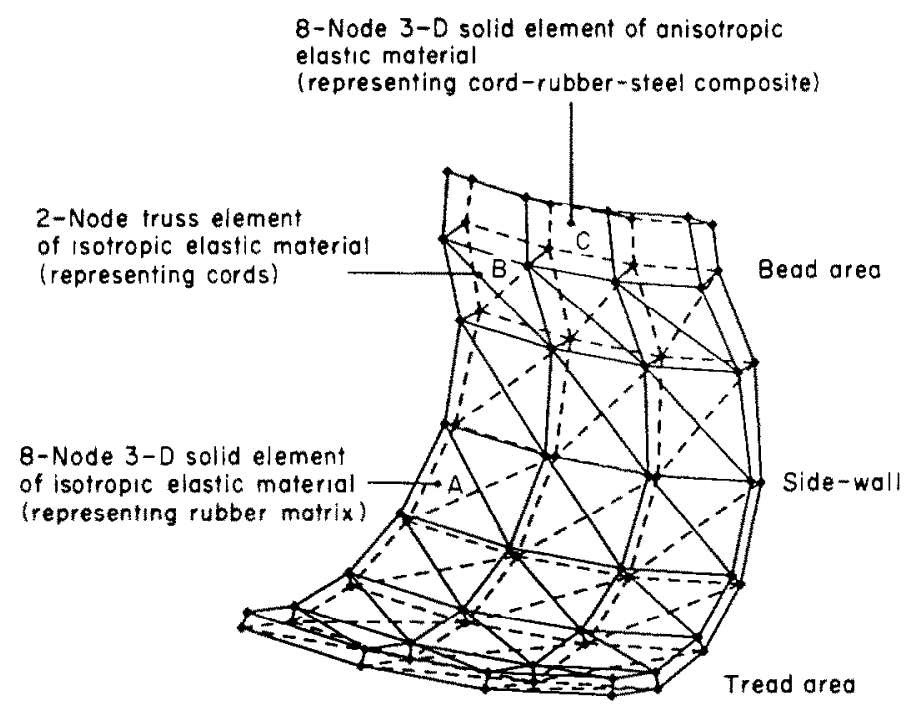

Fig. 1. Finite element model of a bias-ply motorcycle tire, typical section.

actual tires, the number of plies is usually more than two, and the end count of cords is usually near $25 /$ in. Whereas, in the present model one very coarse cord layer (represented by truss elements) is put on the outer surface of the rubber slabs with + beta arrangement and another layer is put on the inner surface of the rubber slabs with - beta arrangement. These two layers represent the numerous cords actually laid in the side-wall and the tread. Accordingly, some of the components in the anisotropic stiffness matrix which contribute to the multi-layer fibrous laminates are inevitably missing and the stress distribution over the cross-section in the model will no doubt be somewhat different from that of the real tire. Fortunately, however, these missing components are not as crucial, the primary characteristics of the bias-ply carcass in terms of load-deformation response are preserved fairly well in spite of their absence.

The present model, has a couple of important merits. The first is, that it preserves the most prominent feature of the cord-rubber laminates by the most straightforward representation using bricks (3-D solid ciements) and strings (truss elements), therefore it is very realistic.

The second merit is that unlike the shell model referred to earlier, there is no need with this model for recalculating the anisotropic material properties. When a tire is inflated by an internal pressure, the cord pantagraph changes its angles to reach the equilibrium configuration. Therefore, if the elastic moduli or the stiffness matrices for the cord-rubber laminates for the state before inflation have been calculated by the technique of micromechanics (equivalent constitutive laws) and is currently being used, they should be up-dated. Furthermore, when the tire contacts the road surface under a certain vertical load, large rotations, thereby appreciable changes in cord orientation occur in the contact area or its vicinity. The geometric nonlinearity due to large rotations can be accounted for by employing the nonlinear strain-displacement relation, however, since the cord orientation is most influential in the equivalent anisotropy of material properties, the anisotropic elastic moduli must be recalculated.
This is a very cumbersome procedure but the present model does not require it, because the brick/truss combination reforms in a build-in fashion the equivalent anisotropic properties. As its converse, if the rotations are not large, the anisotropic material properties calculated at the beginning need not be recalculated. By that reasoning the present model employs linear, anisotropic elastic, 3-D solid elements (marked " $\mathrm{C}$ " in Fig. 1) to represent the bead area where no large rotations, subsequently no large changes in cord orientation are foreseen to occur.

In order to obtain the proper cord orientation, the element size should conform to the cord angle-radius variation relationship given by,

$$
\frac{R_{t}}{\sin \beta_{t}}=\frac{R_{t+1}}{\sin \beta_{t+1}}
$$

where $R_{t}=$ distance of element midpoint from tire axis for $i$ th element; and $\beta_{1}=$ local cord angle for $i$ th element (see Fig. 2); to satisfy the equilibrium contiguration.

Tires under no internal pressure are floppy and can not maintain the same configuration because of residual stresses. Therefore, it is not easy to obtain the value for $R_{t}$ to be used in eqn (1) for uniflated tires in a consistent manner. It is common practice nevertheless, to trace the cross-section from a cutlet of tire. This practice is also followed in the current study.

In the present finite element tire model the local cord angle depends on the aspect ratio of the 3-D solid elements (rubber matrix) because the truss elements (cords) are arranged on the 3-D solid elements diagonally. Therefore, the mesh arrangements along the meridian is not arbitrary but must be made to yield the cord angle variation to conform to the equilibrium configuration, as shown in Fig. 2.

\section{MODEL FOR INFLATION ANALYSIS}

Employing the present finite element model for the tire carcass described above, a half tire model is built for the analysis of deformation under internal pressure loadings. This model represents the left or right semi-sphere of a tire. The nodes in a typical cross- 

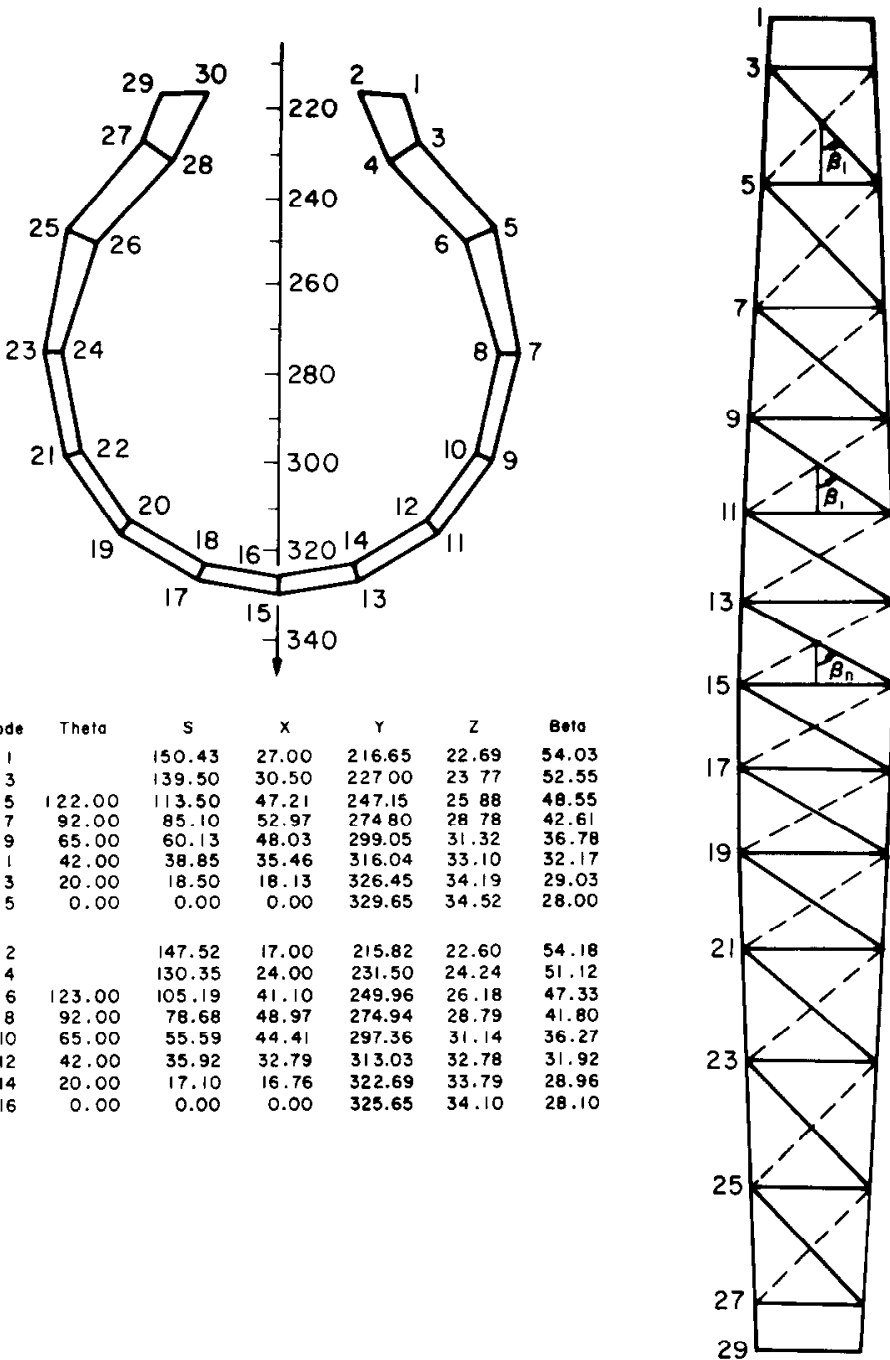

$\begin{array}{crrrrrr}\text { Node } & \text { Theta } & \mathrm{S} & \mathrm{X} & \mathrm{Y} & \mathrm{Z} & \text { Beld } \\ 1 & & 150.43 & 27.00 & 216.65 & 22.69 & \mathbf{5 4 . 0 3} \\ 3 & & 139.50 & 30.50 & 22700 & 2377 & 52.55 \\ 5 & 122.00 & 113.50 & 47.21 & 247.15 & 2588 & 48.55 \\ 7 & 92.00 & 85.10 & 52.97 & 27480 & 2878 & 42.61 \\ 9 & 65.00 & 60.13 & \mathbf{4 8 . 0 3} & 299.05 & 31.32 & 36.78 \\ 11 & 42.00 & 38.85 & \mathbf{3 5 . 4 6} & 316.04 & 33.10 & \mathbf{3 2 . 1 7} \\ 13 & 20.00 & 18.50 & 18.13 & 326.45 & 34.19 & 29.03 \\ 15 & 0.00 & 0.00 & 0.00 & 329.65 & 34.52 & 28.00 \\ & & & & & & \\ 2 & & 147.52 & 17.00 & 215.82 & 22.60 & 54.18 \\ 4 & & 130.35 & 24.00 & 231.50 & 24.24 & 51.12 \\ 6 & 123.00 & 105.19 & 41.10 & 249.96 & 26.18 & 47.33 \\ 8 & 92.00 & 78.68 & 48.97 & 274.94 & 28.79 & 41.80 \\ 10 & 65.00 & 55.59 & 44.41 & 297.36 & 31.14 & 36.27 \\ 12 & 42.00 & 35.92 & 32.79 & 313.03 & 32.78 & 31.92 \\ 14 & 20.00 & 17.10 & 16.76 & 322.69 & 33.79 & 28.96 \\ 16 & 0.00 & 0.00 & 0.00 & 325.65 & 34.10 & 28.10\end{array}$

Fig. 2. Cross section of FE model, truss and 3-D elements.

section are numbered as shown in Fig. 2. For retention of tire at the bead seat of rim, node 3 is supported by a very stiff truss element oriented at an angle of $45^{\circ}$, approximately equal to the normal to the skirt of rim flange. Node 1 is supported by an axially oriented very stiff truss element to allow for radial movements at this node. Nodes 15 and 16 are constrained in the $x$-direction due to symmetry, but are free to move in the $y$-and $z$-directions because of the anisotropy of tire carcass.

\section{MODEL FOR CONTACT DEFORMATION ANALYSIS}

Another half tire model is built for the analysis of deformation under contact loads. This model represents the lower half of a tire and will be called "lower half model" hereafter. The upper half of the tire is assumed to exhibit no deformation, hence eliminated to reduce the degrees of freedom. The nodes lying on the cut-away section are assumed clamped. However, these nodes must be clamped so as to reside at intlation pressure loading position. These nodes would have displaced in the circumferential, radial as well as meridional directions, because of the coupling components in the anisotropic stiffness matrix. These displacements must be obtained using the semi- sphere model prior to the calculation of deformation under the contact loads. The nodes in a typical cross-section are numbered as shown in Fig. 2. In this model the rim retention is achieved simply by claming nodes $1,3,27$ and 29 .

\section{DEFORMATION OF TIRE UNDER INTERNAL PRESSURE}

The deformations of a bias-ply motorcycle tire under internal pressure were calculated using ADINA [R.1]. Figures 3 and 4 show respectively the result of the linear and nonlinear analyses. The calculations were made with the semi-spherical model.

In the original unloaded configuration, the meridional stiffness of this tire at side-wall is smaller than the circumferential stiffness of this tire at its crown, which may explain its odd response characteristics to linear analysis under internal pressure. Its cross-section retracts at the crown and bulges at the side-wall as shown in Fig. 3. However, as soon as incremental loading procedure was employed with the stiffness matrix recalculated in each step, this tendency faded out and a different configuration shown in Fig. 4 was obtained which unquestionably resembles better the real tire deformation.

This is because the cord pantagraph changes its 


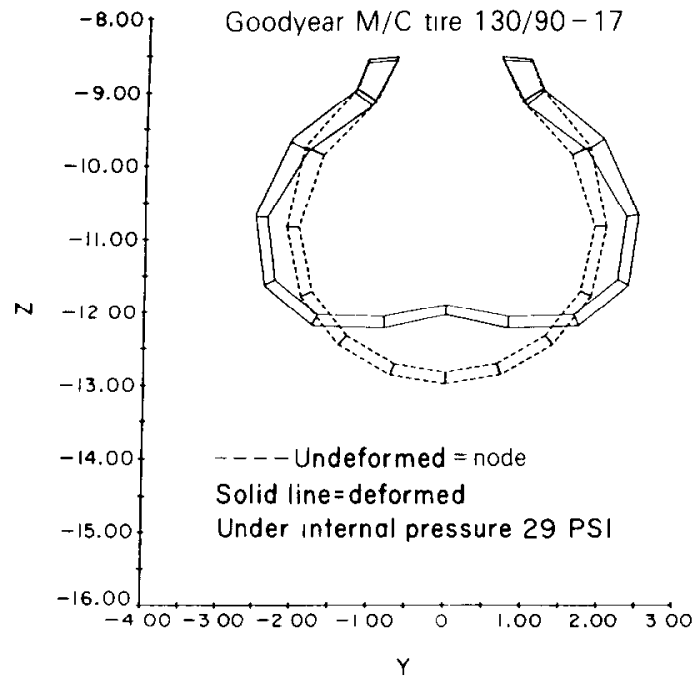

Fig 3. Tire deformation for 29 psi inflation load, lneal analysis

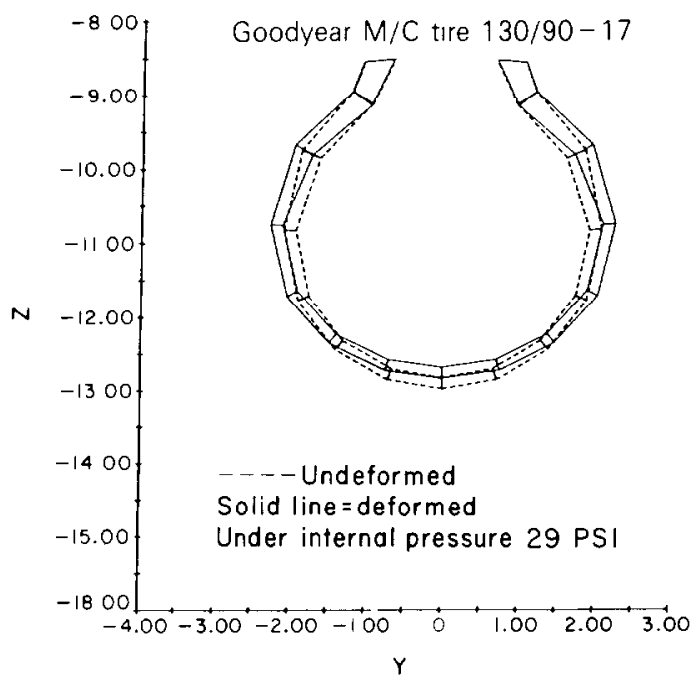

Fig. 4. Tire deformation for 29 psi inflation load, nonlinear analysis.

angle due to the internal pressure in each step, thereby causes appreciable changes in the equivalent anisotropic material properties. Due to the structural feature of the present tire model, no recalculation of anisotropic constitutive laws is necessary. The geometric nonlinearity alone takes care of this problem.

\section{DEFORMATION OF CAMBERED TIRE UNDER VERTICAL LOAD}

The lower-half tire model was inflated to an internal pressure of $2.0 \mathrm{~kg} / \mathrm{cm}^{2}$, was inclined $20^{\circ}$ from the vertical, and was pressed against a rigid flat road surface. Using ADINA, the deformation of this cambered tire model, under static contact loads, was calculated. The frictional coupling between the tire and the road plane was assumed to be negligible until a certain level of normal force was reached, and it stayed at that value when the normal force exceeded that level. A set of prescribed displacements was chosen as input vari- ables. However, since neither the contact forces nor the displacements known a priori, an incremental loading procedure was employed and the prescription for displacements was renewed after each step of calculation.

Figure 5 illustrates the contact logic mechanism employed in this calculation. The initial stage of this tire is depicted by Fig. 5(a). The rim is fixed in space and no contact between the tire and the road plane has been made yet. Figure 5(b) depicts the first step where only node 3 is loaded while other nodes are still free. No adhesion between the tire and the road plane is assumed to occur until a specified level of normal force is reached. After that level of normal force is reached node 3 adheres to the road plane and the tire is further loaded until next contact points 2 and 4 drop pass the road plane as shown in Fig. 5(c).

Knowing the displacements in the previous steps, the prescription for the displacements is renewed. The displacements for nodes 2, 3 and 4 are now prescribed. No transverse constraints, i.e. no adhesion is given to nodes 2 and 4 until a certain level of normal force is reached at these nodes. Then nodes 2 and 4 as well as node 3 adhere to the contact surface and further loads are applied. The process is iterated until the desired loading condition is reached. When a load normal to the road plane is applied, the contact patch spreads and shear as well as normal forces are developed within the contact patch.

Figure 6 shows the outer surface of the tire model, the deformations in an inclined tire under vertical loading for loadstep number 7 . The vectors on the figure indicate the forces exerted by the road plane on the tire.

\section{EVALUATION OF RESULTS}

From the results of the tire model analyses the following observations can be made.

(1) The normal force distribution along the $x$-axis (longitudinal direction) is pyramidal for the early stage of contact (step 1 through step 2 in this case), but soon it becomes trapezoidal (after step 2). As the contact patch spreads out further (after step 4) normal forces at the center of the contact patch decrease in magnitude. The normal force distribution along the

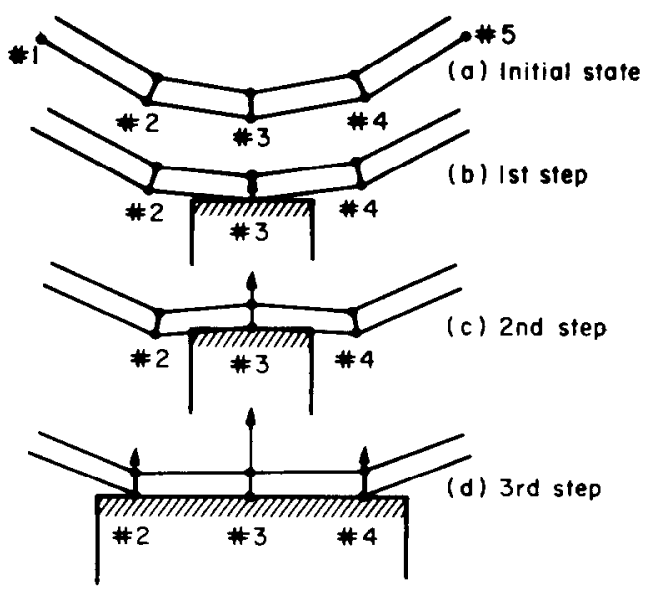

Fig. 5 Logic of contact load mechanısm 
CONTACT FORCES OF A CAMBERED MOTORCYCLE TIRE, GOODYEAR 130/90-17

Load step 7

Vertical deflection $=10.5(\mathrm{~mm})$

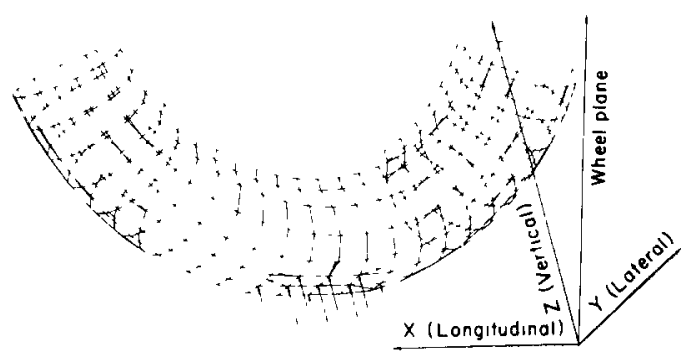

Fig. 6. Contact forces and deformation of a cambered motorcycle tire.

$y$-axis (lateral direction) is also trapezoidal but is weighted more outward (inside the wheel inclination).

(2) The longitudinal forces $F x$ are relatively large along the leading and the trailing edges of the contact patch. They tend to stretch out the contact patch forwards and rearwards, respectively. The longitudinal forces $F x$ seem to dominate the lateral forces Fy. However, the longitudinal forces on the whole distribute rather symmetrically, thus balance each other and produce a resultant of trivial magnitude.

(3) In contrast to the above the distribution of the lateral forces $F y$ is noticeably asymmetric. Lateral forces of appreciable magnitude are produced acting on the outer edge of the contact patch, but along its inner edge relatively small lateral forces are produced. Therefore, the resultant lateral force has an appreciable magnitude and acts to pull the tire inside the wheel inclination.

(4) Since the centroid of normal forces for an inclined tire does not coincide with the center of tire contact (center of tire contact is defined as the interscction of wheel plane and the vertical projection of the spin axis of the wheel onto the road plane), moments are produced about $x$ - and $y$-axes. The moment about $y$-axis is called "rolling resistance moment", and the moment about the $x$-axis $M x$ is called "overturning moment". The sense of overturning moment is defined to be positive clockwise when viewed in the positive direction of the $x$-axis. The overturning moment acts against the inclination of wheel and increases with vertical load.

(5) The longitudinal forces $F x$ and the lateral forces Fy cause a moment which tends to rotate the tire about the $z$-axis as the resultants of these forces do not pass through the center of tire contact. This moment is called "aligning torque" $M z$ and its sense is defined to be positive clockwise when viewed in the positive direction of the $z$-axis. The aligning torque for an inclined tire increases and changes sign, as the vertical load is increased. It shows a rapid build-up after the vertical load reaches a certain level in a direction that tends to rotate the front of the tire outward (inside the inclination of wheel).

Table 1 shows the values of the forces $F x$ (longltudinal), $F y$ (lateral), $F_{z}$ (normal), and the moments $M x$ (overturning) and $M z$ (aligning torque) tabulated for each step of the incremental vertical loading. The individual contribution of forces $F x$ and $F_{y}$ to the aligning torque $M z$ are shown listed in Table 2. Table 1 listing is shown plotted in Fig. 7. For a contact

Table 1. Contact force and moment components of a $20^{\circ}$-camber motorcycle tire (Goodyear 130/90-17)

\begin{tabular}{ccccccc}
\hline $\begin{array}{c}\text { Load } \\
\text { step }\end{array}$ & $\begin{array}{c}\text { Vertical } \\
\text { deflection } \\
\text { DELZ[MM] }\end{array}$ & $\begin{array}{c}\text { Longitudinal } \\
\text { force } \\
\text { Fx[KG] }\end{array}$ & $\begin{array}{c}\text { Lateral } \\
\text { force } \\
\text { Fy[KG] }\end{array}$ & $\begin{array}{c}\text { Vertical } \\
\text { force } \\
F z[K G]\end{array}$ & $\begin{array}{c}\text { Overturning } \\
\text { moment } \\
M x[K G-M M]\end{array}$ & $\begin{array}{c}\text { Aligning } \\
\text { torque } \\
M z[K G-M M]\end{array}$ \\
\hline 0 & 0.0 & 0.0 & 0.0 & 0.0 & 0.0 & 0.0 \\
1 & 1.5 & -0.03 & 0.01 & -9.60 & -178.45 & 0.11 \\
2 & 2.7 & -0.59 & 2.85 & -44.16 & -811.99 & 10.84 \\
3 & 4.1 & 1.28 & 12.41 & -92.01 & -1942.56 & -45.83 \\
4 & 5.3 & 3.26 & 23.95 & -129.38 & -3236.43 & -96.06 \\
5 & 7.3 & -5.67 & 33.89 & -178.16 & -4212.20 & 316.89 \\
6 & 9.5 & -15.99 & 41.86 & -230.55 & -5015.99 & 889.77 \\
\hline
\end{tabular}

Table 2. Breakdown of aligning torque $M z$ in Table 1, into components due to forces $F x$ and $F y$

\begin{tabular}{ccccc}
\hline $\begin{array}{c}\text { Load } \\
\text { step }\end{array}$ & $\begin{array}{c}\text { Vertical } \\
\text { deflection } \\
\text { DELZ[MM] }\end{array}$ & $\begin{array}{c}\text { Total } \\
M z[\text { total] } \\
{[\mathrm{KG}-\mathrm{MM}]}\end{array}$ & $\begin{array}{c}\text { Self-aligning torque } \\
\text { Due to } F x \\
M z[F X] \\
{[\mathrm{KG}-\mathrm{MM}]}\end{array}$ & $\begin{array}{c}\text { Due to } F y \\
M z\left[F y^{\prime}\right] \\
{[\mathrm{KG}-\mathrm{MM}]}\end{array}$ \\
\hline 0 & 0.0 & 0.0 & 0.0 & 0.0 \\
1 & 1.5 & 0.11 & 0.73 & -0.61 \\
2 & 2.7 & 10.84 & 8.15 & 2.68 \\
3 & 4.1 & -45.83 & -25.02 & -20.81 \\
4 & 5.3 & -96.06 & 26.72 & -122.78 \\
5 & 7.3 & 316.89 & 353.53 & -36.64 \\
6 & 9.5 & 889.77 & 768.88 & 120.89 \\
\hline
\end{tabular}




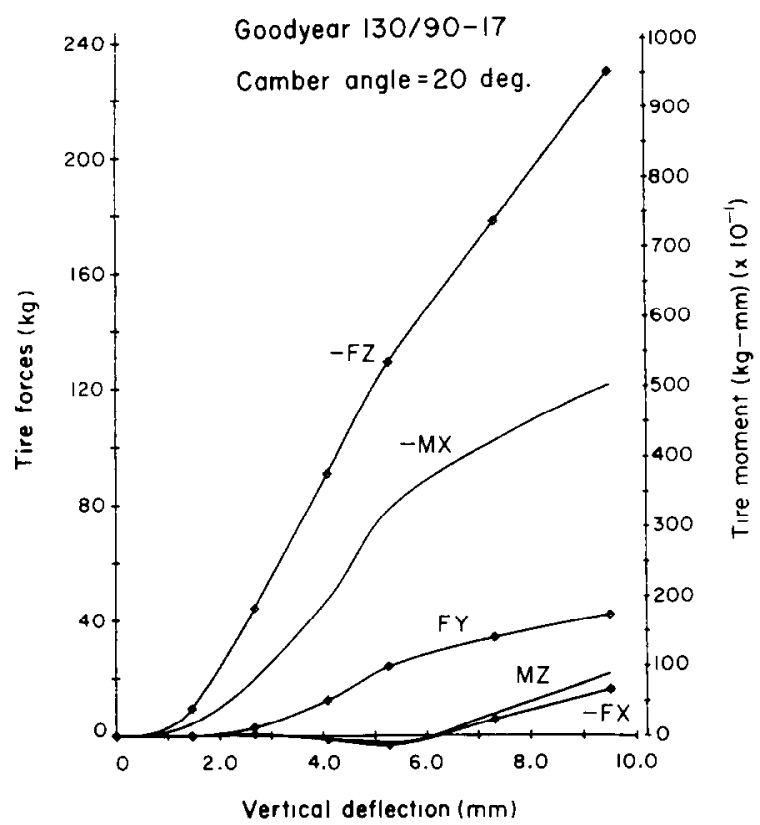

Fig. 7. Contact forces and moments on a cambered motor cycle tire.
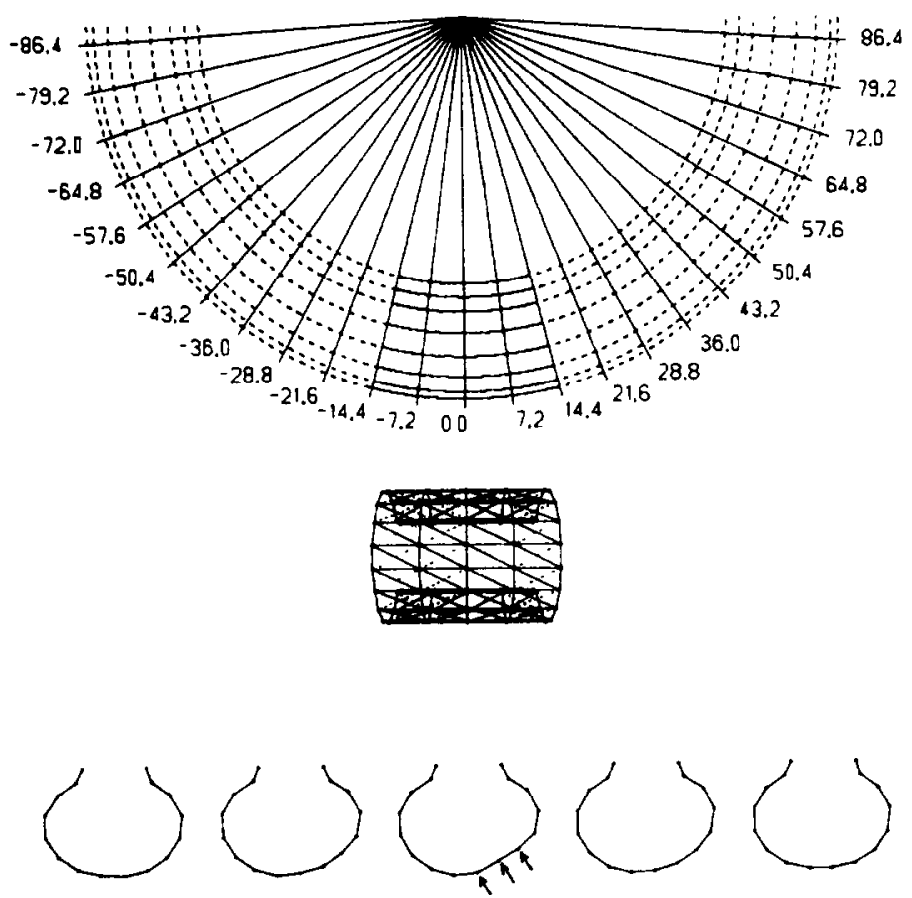

Fig. 8. Deformation at various sections along cambered tire from contact patch load.

patch load on a lower-half tire model Fig. 8 shows the deformations in the cross-section at various locations along the tire.

In conclusion, the authors feel encouraged with the results they obtained using the ADINA program and are looking forward to comparing them with experi- mental work currently in progress at the University of Michigan.

\section{REFERENCE}

1. K.-J. Bathe, ADINA - a finite element program for automatic dyanmic incremental nonlinear analysis. Rep. 82448-1, MIT (1978). 